\title{
Comportamiento de picadura de Anopheles darlingi Root, 1926 (Diptera: Culicidae) y su asociación con la transmisión de malaria en Villavicencio (Colombia)
}

\author{
Martha Liliana Ahumada', Paula Ximena Pareja', Luz Stella Buitrago², Martha L. Quiñones ${ }^{3}$ \\ 1 Laboratorio de Entomología, Instituto Nacional de Salud, Bogotá, D.C., Colombia \\ 2 Secretaría Seccional de Salud del Meta, Villavicencio, Colombia \\ 3 Departamento de Salud Pública, Universidad Nacional de Colombia, Bogotá, D.C., Colombia
}

Introducción. Anopheles darlingi es el principal vector de la malaria, o paludismo, en el neotrópico. Esta especie es reconocida por presentar un comportamiento antropofílico y variabilidad en sus hábitos de picadura a lo largo de su rango de distribución.

Objetivo. Caracterizar el comportamiento de picadura de An. darlingi y establecer su relación con la transmisión de la malaria en Villavicencio.

Materiales y métodos. Entre 2008 y 2009 se llevaron a cabo un estudio de tipo transversal y uno longitudinal, en cinco localidades de Villavicencio con transmisión de malaria. Estos incluyeron recolección de ejemplares inmaduros y de adultos en las viviendas. Se utilizó la prueba ELISA para la detección de infección con Plasmodium spp.

Resultados. Se recolectaron 2.772 mosquitos. Anopheles darlingi fue la especie predominante en las capturas con atrayente humano. Los criaderos identificados para esta especie fueron pantanos, caños, lagunas y estanques piscícolas. Anopheles darlingi estuvo presente durante todo el año, con densidades mensuales promedio entre 2,2 y 55,5 mosquitos por persona por noche. Presentó actividad hematofágica durante toda la noche en el intradomicilio y en el peridomicilio. De las 18:00 a las 22:00, se registraron entre el $47 \%$ y el $81 \%$ de los mosquitos capturados en 12 horas de observación. Se encontró una tasa de infección con Plasmodium falciparum de 0,05 \% y se estimó una tasa entomológica de inoculación de 2,9 picaduras infecciosas por persona al año.

Conclusión. Anopheles darlingi se encontró infectado con P. falciparum, estuvo presente durante todo el año y exhibió características en su comportamiento de picadura que favorecen el contacto entre humano y vector, lo cual es un riesgo permanente para la transmisión de la malaria en Villavicencio.

Palabras clave: Anopheles, conducta animal, insectos vectores, Plasmodium, malaria, Colombia. doi: http://dx.doi.org/10.7705/biomedica.v33i2.1492

Biting behavior of Anopheles darlingi Root, 1926 (Diptera: Culicidae) and its association with malaria transmission in Villavicencio (Meta, Colombia)

Introduction: Anopheles darlingi is the main malaria vector in the neotropics. This species is recognized by its anthropophilic behavior and its high variability in biting activity throughout its distribution range.

Objective: To characterize the biting behavior of An. darlingi and to establish its association with malaria transmission in Villavicencio.

Materials and methods: Between 2008 and 2009, a cross sectional and a longitudinal entomological study were carried out in 5 localities with malaria transmission in Villavicencio. Mosquito collections included breeding sites search and human landing catches in houses. Collected mosquitoes were analyzed for Plasmodium using the ELISA standard protocol.

Results: A total of 2,772 mosquitoes were collected in the study. Anopheles darlingi was the most abundant anopheline species. The most common breeding sites for this species were marshes, streams, lakes and fish ponds. Anopheles darlingi was found at all times during the year with monthly average human biting rates between 2.2 y 55.5 mosquitos/person/night. This species was collected throughout the night, indoors and outdoors, and 47 to $81 \%$ of $A n$. darling captured during twelve hours of observation (18:00 to 06:00) were collected between18:00 and 22:00. Anopheles darlingi was found

\section{Contribución de los autores:}

Martha Liliana Ahumada: realización del trabajo de campo y laboratorio, análisis de datos y elaboración del manuscrito.

Paula Ximena Pareja y Luz Stella Buitrago: recolección e identificación de material entomológico y apoyo en la realización de las series entomológicas.

Martha Lucía Quiñones: orientación del trabajo, participación en el análisis de datos, elaboración y revisión del manuscrito. 
positive for $P$. falciparum with a $0.05 \%$ rate and the entomological inoculation rate was estimated at 2.9 infective bites/person per year.

Conclusion: Anopheles darlingi was infected with $P$. falciparum, it was found all year long and it exhibited characteristics in biting behavior that favor human-vector contact, being a permanent risk for malaria transmission in Villavicencio.

Key words: Anopheles; behavior, animal; insect vectors, Plasmodium, malaria, Colombia.

doi: http://dx.doi.org/10.7705/biomedica.v33i2.1492

Anopheles darlingi Root 1926 es el principal vector de malaria en la región neotropical, dada la evidencia epidemiológica, los hallazgos de infección con Plasmodium falciparum y $P$. vivax, y su asociación con los humanos (1-4). Una de las características que hace de esta especie el vector más eficiente de los parásitos de malaria en las Américas, es su comportamiento altamente antropofílico, con actividad tanto endofágica como exofágica $(5,6)$.

Varios trabajos realizados en Latinoamérica sobre el comportamiento de An. darlingi, han demostrado que presenta patrones estacionales de picadura que dependen de la localidad y la estación climática en la que se hace la recolección de los insectos $(1,6-8)$. Se ha establecido que presenta mayor densidad en los meses lluviosos y menor en los meses más secos (9-11), aunque se identifica, en algunas áreas, la importancia de los periodos de transición entre las estaciones climáticas (12). Sin embargo, los estudios han establecido que no es significativa la asociación entre la densidad de los mosquitos capturados con atrayente humano y los niveles de precipitación $(1,11,12)$. En Villavicencio, en la década de los 40, la detección de infección con especies de Plasmodium permitió establecer que la habilidad de An. darlingi como vector disminuía después de finalizar la estación lluviosa $(13,14)$.

Con relación al comportamiento horario de picadura, An. darlingi presenta gran variabilidad según su rango de distribución (15). Se caracteriza por estar presente durante toda la noche, aunque exhibe diferentes patrones que incluyen un máximo de actividad al atardecer, a la medianoche o al amanecer $(1,2,7,8,11,16)$. En Colombia se ha observado que An. darlingi presenta una mayor actividad entre las 18:00 y las 19:00, las 21:00 y las 24:00 y las 05:00 y las 06:00 horas (17-19).

\footnotetext{
Correspondencia:

Martha Liliana Ahumada, Laboratorio de Entomología, Instituto Nacional de Salud, Avenida calle 26 № 51-20, Bogotá, D.C., Colombia.

Teléfono: (051) 220 7700, extensiones 1341 y 1343

mahumada@ins.gov.co

Recibido: 01/02/12; aceptado:06/12/12
}

Villavicencio pertenece a la región de la Orinoquia, la cual ocupa el tercer lugar en número de casos de paludismo en Colombia con un índice parasitario anual (IPA) de 6,8 por 1.000 habitantes $(20,21)$. En este municipio se presenta transmisión variable, endémica y epidémica, que es afectada por el desplazamiento de la población vulnerable y de portadores de parásitos. Entre 2000 y 2010 , se reportaron al año entre 60 y 300 casos, con predominio de $P$. vivax (>70 \%) y con un IPA inferior a 1,0 , lo cual clasifica a este municipio como de bajo riesgo para la transmisión de la malaria (22). Sin embargo, aunque en este municipio no se registra un mayor número de casos de malaria, la transmisión se caracteriza por estar localizada en zonas periurbanas, evento que ha sido considerado como un problema de salud pública dado el riesgo de urbanización de la enfermedad $(23,24)$. Esta transmisión periurbana ha sido favorecida por las condiciones ambientales y de desarrollo urbano, que han permitido la creación de ambientes propicios para la permanencia y proliferación de los vectores en Villavicencio, muy particularmente de An. darlingi, que en bajas densidades es capaz de mantener la transmisión del paludismo $(15,18,25)$.

El presente estudio se hizo con el propósito de caracterizar el comportamiento de picadura de $A n$. darlingiy establecer su asociación con la transmisión de la malaria en el municipio de Villavicencio. Esta información contribuirá al entendimiento de la dinámica de transmisión, aspecto indispensable para la selección de las medidas de control vectorial adecuadas en el área.

\section{Materiales y métodos \\ Áreas de estudio}

Villavicencio es un municipio del departamento del Meta, localizado a los $4^{\circ} 09^{\prime} 12^{\prime \prime}$ de latitud norte y 7338'06" de longitud oeste, a una altura de 467 msnm; presenta temperatura promedio de $25,5^{\circ} \mathrm{C}$, precipitación media anual de $3.663 \mathrm{~mm}$ y humedad relativa promedio anual del $80 \%$. Se registra un régimen de lluvias monomodal, en el cual los meses más lluviosos son abril, mayo y octubre (26). Las localidades del estudio, Barcelona (4²'22"; 
$\left.73^{\circ} 21^{\prime} 51^{\prime \prime}\right)$, Quintas de San Fernando (46'60";

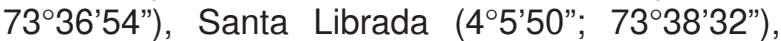

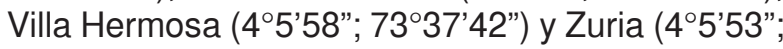
$\left.73^{\circ} 37^{\prime} 49^{\prime \prime}\right)$, se localizan en la periferia de esta ciudad. Se caracterizan por la presencia de asentamientos humanos rodeados por grandes extensiones de pastizales con áreas inundables, caños y relictos de bosques intervenidos. Es un área receptora de migrantes, quienes en ocasiones pueden provenir de zonas con transmisión de malaria, dado que el municipio proporciona ventajas sociales, económicas y culturales propias de un centro urbano en desarrollo (27).

\section{Diseño del estudio}

Para identificar las especies de Anopheles presentes en las áreas de transmisión de malaria del municipio de Villavicencio y establecer patrones de comportamiento de picadura con respecto al domicilio humano, se llevaron cabo dos estudios. El primero fue de tipo transversal, el cual incluyó recolección de adultos con atrayente humano y captura de ejemplares inmaduros en los sitios de cría encontrados en las cinco localidades antes mencionadas, donde se había registrado, por lo menos, un caso de malaria durante el 2008. Las capturas de los mosquitos se hicieron en junio, julio y septiembre de 2008, entre 5 y 12 noches por localidad, en las viviendas donde se presentaron casos. El segundo estudio, de tipo longitudinal, se llevó a cabo entre julio de 2008 y junio de 2009 en Quintas de San Fernando, localidad que registró un mayor número de casos de malaria durante el primer semestre de 2008. Las capturas de adultos se hicieron en una única vivienda, de 3 a 4 días cada mes, durante 10 meses, siendo la misma durante el estudio. Los datos de precipitación fueron medidos diariamente y la temperatura se registró a intervalos de 15 minutos utilizando un datalogger (Extech Instruments ${ }^{\mathrm{TM}}$, referencia 42270). Los instrumentos de medición fueron instalados en esta vereda.

\section{Recolección de mosquitos adultos}

En el estudio longitudinal, los mosquitos adultos se capturaron con atrayente humano protegido, simultáneamente en el intradomicilio y el peridomicilio entre las 18:00 y las 06:00, con rotación de los recolectores cada hora entre los dos sitios antes mencionados. En el caso del estudio transversal, las capturas se hicieron entre las 18:00 y las 23:00. Estas se efectuaron durante los primeros 50 minutos de cada hora. Los mosquitos se colocaron en frascos rotulados, especificando el lugar (intradomicilio o peridomicilio), fecha, hora, nombre del recolector y número de mosquitos. Después de haberlos sacrificado utilizando trietilamina, se empacaron individualmente en viales Eppendorf $^{\text {TM }}$ de $1,5 \mathrm{ml}$ con la tapa perforada. Los tubos se colocaron en bolsas de cierre hermético que contenían sílica-gel para la preservación del material entomológico. Las capturas las llevaron a cabo personal entrenado de la Secretaría de Salud del departamento del Meta y del Laboratorio de Entomología del Instituto Nacional de Salud, previa firma de un consentimiento informado.

\section{Recolección de ejemplares inmaduros}

Para la identificación de los sitios de cría, se buscaron formas inmaduras alrededor de las viviendas seleccionadas para capturarlas con atrayente humano. Las larvas se recolectaron con cucharones, haciendo 10 tomas por metro cuadrado. Posteriormente, las larvas se empacaron en vasos plásticos y se transportaron al laboratorio de la Secretaría de Salud del departamento del Meta y al Laboratorio de Entomología del Instituto Nacional de Salud para la obtención de las series entomológicas (cría de larvas hasta la obtención de mosquitos adultos). Para cada uno de los sitios de cría revisados, se estableció el tipo de criadero, la presencia de vegetación y el porcentaje de sombra sobre el área total del espejo de agua.

\section{Determinación taxonómica}

La determinación taxonómica de los ejemplares, adultos e inmaduros, se hizo utilizando claves dicotómicas (28-31).

\section{Detección de infección natural con Plasmodium spp.}

Después de realizar la determinación taxonómica de los mosquitos adultos recolectados con atrayente humano, se tomó la cabeza y el tórax de cada mosquito y se procesaron conjuntamente con el fin de detectar en estas partes la proteína del circumsporozoíto de $P$. falciparum, $P$. vivax VK210 y $P$. vivax VK247. Se siguió el protocolo estandarizado $(32,33)$ que utiliza el kit de la prueba de ELISA (Enzyme-Linked ImmunoSorbent Assay), distribuido por los Centers for Disease Control and Prevention (CDC, Atlanta, Estados Unidos).

\section{Variables estudiadas y análisis de datos}

La densidad mensual y la actividad horaria de An. darlingi se expresaron como el promedio geométrico del número de mosquitos recolectados por persona por noche y mosquitos por persona por hora, respectivamente. La infección natural se 
calculó como el porcentaje de mosquitos infectados con parásitos de Plasmodium spp. para cada una de las especies de Anopheles encontradas. La tasa de inoculación entomológica (TIE), definida como el número de picaduras infecciosas que puede recibir una persona durante un año, se calculó utilizando la siguiente expresión: $\mathrm{TIE}=\mathrm{ma} \times$ te, donde ma es la tasa de picadura (mosquitos por persona por noche) por 365 días del año, y te es la tasa de infección de los mosquitos con esporozoítos de Plasmodium (34).

Para identificar los periodos de mayor exposición que pudieran tener los humanos a las picaduras de An. darlingi durante la noche, se establecieron tres lapsos de tiempo de acuerdo con los hábitos observados en la población: periodo 1 (P1), entre las 18:00 y las 22:00 (cuatro horas); periodo 2 (P2), entre las 22:00 y las 04:00 (seis horas) y, periodo 3 (P3), entre las 04:00 y las 06:00 (tres horas). Para cada uno se calculó la contribución relativa como el porcentaje de mosquitos recolectados por periodo con respecto al total de los mosquitos capturados entre las 18:00 y las 06:00.

Para la comparación de las variables densidad y contribución relativa, registradas para An. darlingi, se utilizaron las pruebas de Mann-Whitney y Kruskal-Wallis. Se consideró significativo un valor de $p$ menor de 0,05. El análisis estadístico se hizo con el programa SPSS ${ }^{\text {TM }}$ (Statistical Package for the Social Sciences), versión 19.0.

\section{Consideraciones éticas}

Las metodologías empleadas para la recolección de los mosquitos fueron aprobadas por el Comité de Ética del Instituto Nacional de Salud y de la Universidad Nacional de Colombia, sede Bogotá.

\section{Resultados}

\section{Especies recolectadas del género Anopheles}

En las cinco localidades estudiadas se recolectaron 2.772 mosquitos con atrayente humano protegido durante 56 noches. Anopheles darlingi $(n=2.656)$ fue la especie predominante. Se capturó junto con An. albitarsis s.I. $(\mathrm{n}=74)$, An. rangeli $(\mathrm{n}=19)$, An. braziliensis $(n=14)$, An. costai $(n=8)$ y $A n$. triannulatus s.l. $(n=1)$. Del material entomológico recolectado en 30 sitios de cría, se obtuvieron 193 series entomológicas, de las cuales, 83 fueron de An. darlingi. Esta especie se capturó junto con An. albitarsis s.I. $(\mathrm{n}=37)$ y An. rangeli $(\mathrm{n}=66)$. Las larvas de An. darlingi se encontraron en 10 de los 30 criaderos examinados, distribuidas de la siguiente manera: en criaderos del tipo pantano, el cual incluyó potreros inundados temporalmente, $39 \%$; caños, $38 \%$; lagunas, 8,2 \%; estanques piscícolas, 7,5\%, y pozos, 6,8 \%. Los sitios de cría en donde de identificó esta especie presentaron un porcentaje de sombra sobre el espejo de agua que osciló entre 0 y $83 \%$, con presencia de vegetación circundante (10 de 10 criaderos positivos), emergente (8 de 10 criaderos positivos) y flotante (6 de 10 criaderos positivos).

\section{Actividad de picadura, variación mensual de la densidad y relación con la transmisión de la malaria}

En el estudio longitudinal en Quintas de San Fernando, vereda Apiay, entre julio de 2008 y junio de 2009, se efectuaron capturas con atrayente humano durante 36 noches distribuidas en 10 meses de observación (no se hicieron capturas en enero y marzo de 2009). Durante este periodo se recolectaron 1.570 mosquitos, a partir de los cuales se identificaron seis especies: An. darlingi $(\mathrm{n}=1.479)$, An. albitarsis s.I. $(\mathrm{n}=68)$, An. braziliensis $(n=13)$, An. rangeli $(n=7)$, An. costai $(n=2)$, y $A n$. triannulatus s.l. $(\mathrm{n}=1)$; An. darlingi fue la especie más abundante, con $94 \%$ del total de mosquitos capturados.

Las observaciones hechas en diez meses, permitieron establecer que An. darlingihabía estado presente durante todo el año con comportamiento endofágico y exofágico, dado que fue capturado en el intradomicilio y en el peridomicilio, con 608 y 797 mosquitos recolectados, respectivamente. Se determinó que presentó actividad hematofágica durante toda la noche en el intradomicilio y el peridomicilio, con un máximo entre las18:00 y las 19:00 y un posterior descenso gradual a lo largo de la noche (figura 1). Para este municipio se obtuvo una tasa de picadura promedio de 17 picaduras por persona por noche, con valores que oscilaron entre 2,0 y 76,0 mosquitos por persona por noche en el intradomicilio y entre 1,7 y 89,0 mosquitos por persona por noche en el peridomicilio. No se presentó diferencia significativa entre la densidad obtenida en el intradomicilio y en el peridomicilio para cada uno de los meses de observación (prueba de Mann-Whitney: $W=47 ; p=0,59$; nivel de confianza $95 \%$ ) (figura 2).

Con relación a la variación mensual de la densidad, se observó una diferencia significativa entre la densidad registrada durante el mes de febrero, comparada con los otros meses de estudio $\left(\chi^{2}=15,315 ; p<0,001\right)$. Lo anterior permitió 


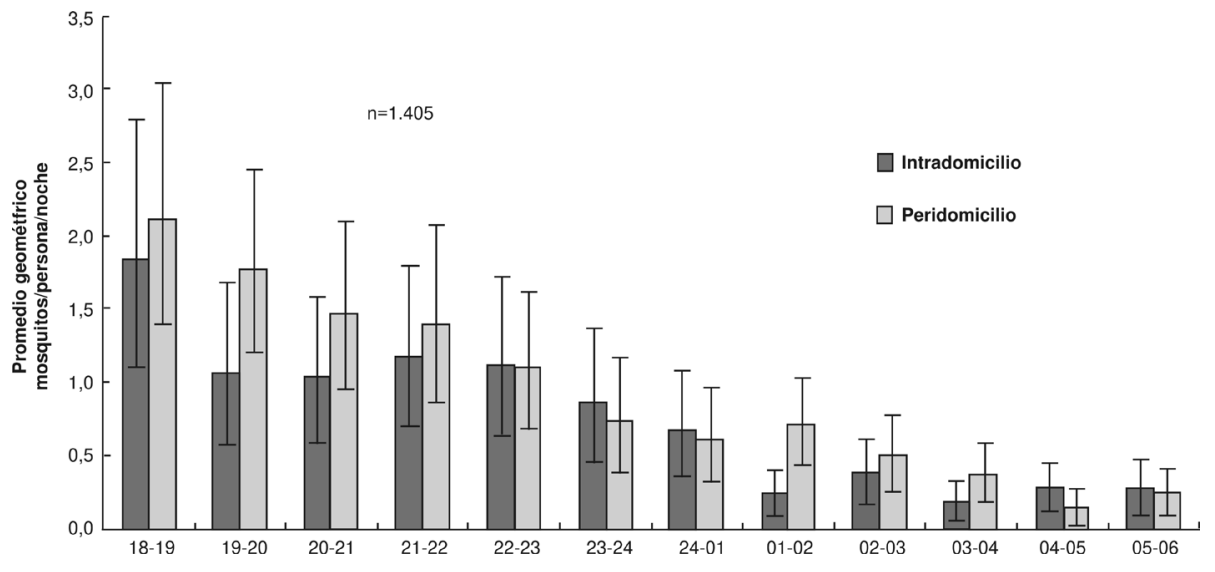

Figura 1. Actividad horaria de picadura de Anopheles darlingien Quintas de San Fernando, vereda Apiay, municipio de Villavicencio, entre julio de 2008 y junio de 2009 (promedio geométrico \pm intervalo de confianza $95 \%$ ).

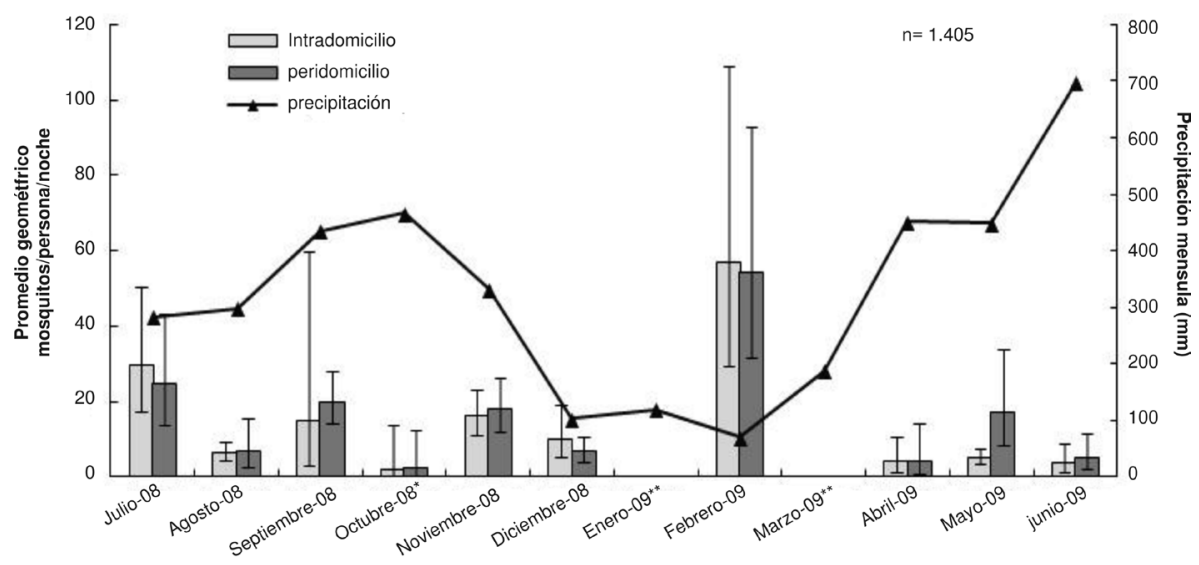

Figura 2. Comportamiento de la densidad mensual de Anopheles darlingi y la precipitación registrada por la Secretaría de Salud del Meta entre julio de 2008 y junio de 2009 en la estación de Apiay, Villavicencio (promedio geométrico \pm intervalo de confianza $95 \%)$.

* Se capturaron mosquitos entre las 18:00 y las 00:00.

${ }^{* *}$ No se capturaron mosquitos.

establecer que An. darlingi estuvo presente durante todo el año, con densidades mensuales promedio que oscilaron entre 2,2 y 55,5 mosquitos por persona por noche (figura 3), donde el mayor valor ocurrió en febrero, cuando se registró una menor precipitación ( $<100 \mathrm{~mm}$ ) (figura 2) y un mayor promedio de temperatura mensual $\left(27,3 \pm 0,13^{\circ} \mathrm{C}\right)$ (no se presentan los datos). Durante los otros meses de estudio, la densidad estuvo por debajo de 27,1 mosquitos por persona por noche.

Con respecto a la transmisión de la malaria durante 2008 y 2009, se observó aumento en el número de casos en julio y septiembre de 2008 y febrero de 2009 , meses en que se registró un incremento en la densidad de An. darlingi (figura 3).

\section{Riesgo de exposición de la población humana a picaduras de Anopheles darlingi}

Los períodos de exposición del humano a la picadura del vector durante la noche se presentan en la figura 4. En el intradomicilio se observó que: durante el P1, los porcentajes de contribución oscilaron entre 38,9 y $78,3 \%$, con un promedio de $57 \%$; durante el P2, oscilaron entre 20,5 y $52,9 \%$ con un promedio de $37 \%$, y durante el P3 oscilaron entre 0 y $16,7 \%$ con un promedio de $6,4 \%$. Además, en el intradomicilio hubo una mayor actividad en el P1 durante 2008, cuando los porcentajes de contribución estuvieron por encima de $64 \%$, comparado con el 2009 cuando la actividad de An. darlingi disminuyó. En el peridomicilio se observó que: durante el P1, los 


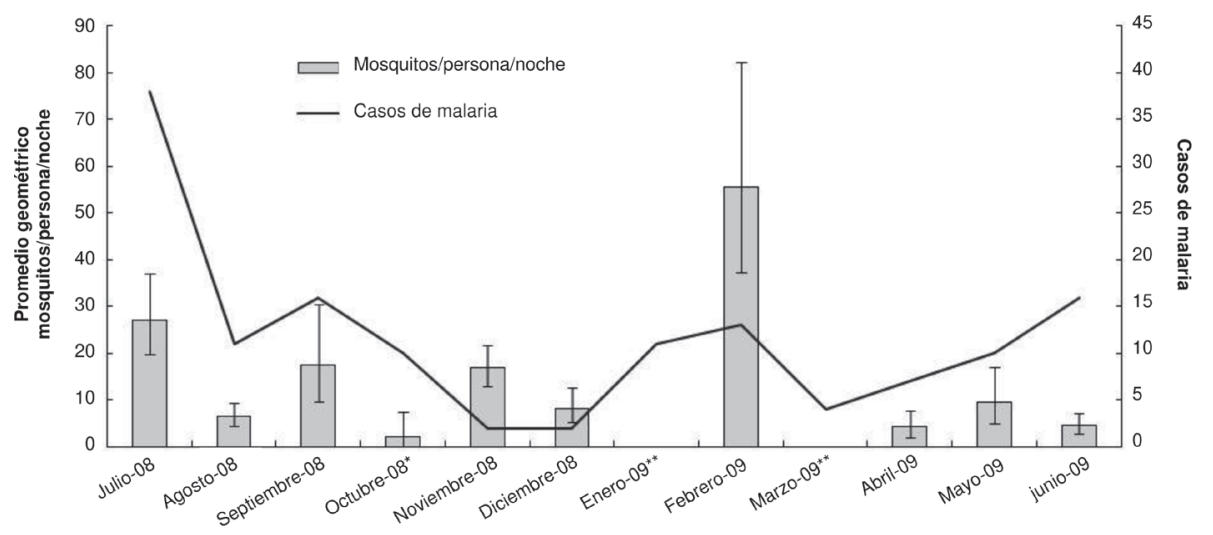

Figura 3. Comportamiento de la densidad de Anopheles darlingi entre julio de 2008 y junio de 2009 (promedio geométrico \pm intervalo de confianza $95 \%$ ) y su relación con la transmisión de la malaria)

Fuente: SIVIGILA, Instituto Nacional de Salud

* Se capturaron mosquitos entre las 18:00 y 00:00.

${ }^{* *}$ No se capturaron mosquitos.
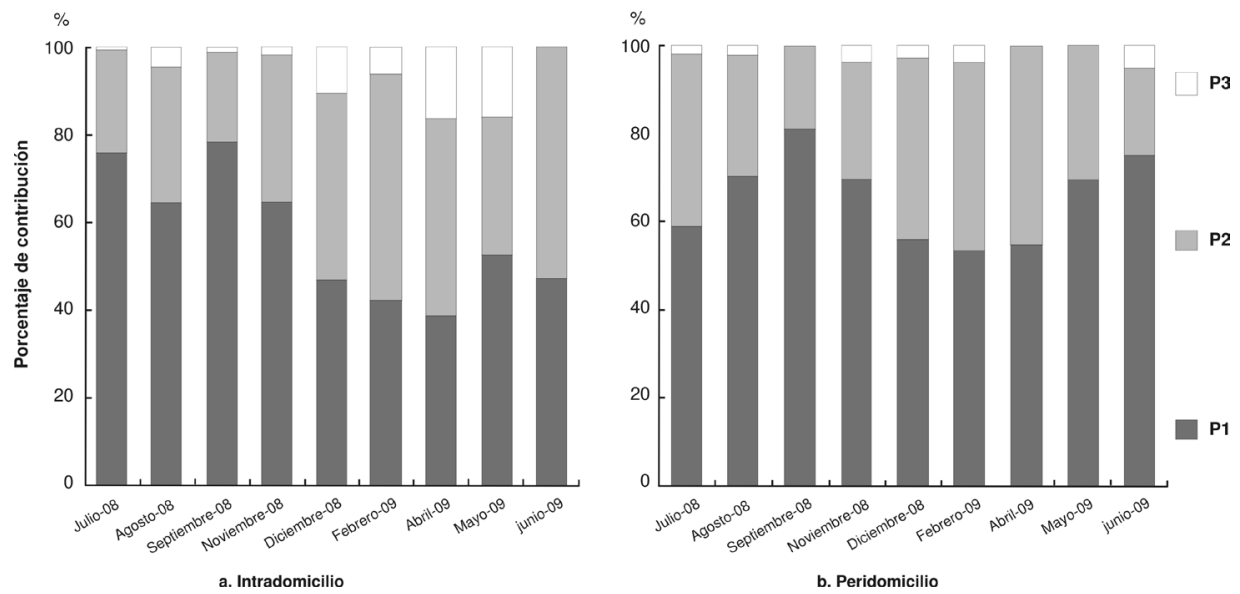

Figura 4. Actividad de picadura de Anopheles darlingi expresada como contribución relativa durante tres periodos de la noche. P1:18:00 a 22:00; P2: 22:00 a 04:00; P3: 04:00 a 06:00 (no se incluye octubre de 2008 porque no se hicieron capturas durante 12 horas).

porcentajes de contribución oscilaron entre 53,3 y $81 \%$ con un promedio de 65,3\%; durante el P2, oscilaron entre 19 y $45,5 \%$ con un promedio de 33 $\%$, y durante el P3, oscilaron entre 0 y $5,0 \%$ con un promedio de $2,2 \%$.

No se observaron diferencias significativas entre los porcentajes de contribución obtenidos en el intradomicilio y el peridomicilio para cada uno de los periodos, $\mathrm{P} 1$ ( $\left.\chi^{2}=2,123 ; p=0,145\right), \mathrm{P} 2\left(\chi^{2}=1,031\right.$; $p=0,310)$ y $P 3\left(\chi^{2}=1,773 ; p=0,183\right)$. Al comparar entre los valores obtenidos para cada uno de los periodos en cada uno de los sitios de muestreo, se estableció que hubo diferencia significativa entre los periodos en el intradomicilio $\left(\chi^{2}=20,025\right.$; $p>0,001)$ y el peridomicilio $\left(\chi^{2}=23,171 ; p>0,001\right)$.

\section{Infección natural con especies de Plasmodium spp. y tasa de inoculación entomológica}

Se procesaron 2.208 mosquitos para la detección de infección natural con $P$. falciparum, $P$. vivax VK247 y $P$. vivax VK210, correspondientes a An. darlingi $(\mathrm{n}=2.125)$, An. albitarsis s.l. $(\mathrm{n}=51)$, An. rangeli $(\mathrm{n}=12)$, An. triannulatus s.I. $(\mathrm{n}=1)$, An. braziliensis $(\mathrm{n}=11)$ y An. costai $(\mathrm{n}=8)$. En el municipio de Villavicencio se encontró infectado un ejemplar de An. darlingi (1/2.125) con P. falciparum, para una tasa de infección de 0,05\%. El mosquito infectado fue capturado en la localidad de Santa Librada en septiembre de 2008 entre las 18:00 y las 19:00 en el intradomicilio, intervalo de tiempo que hace parte del P1. 
La tasa de inoculación entomológica se estimó a partir del valor de densidad de 17 picaduras por persona por noche y de la tasa de infección por $P$. falciparum de 0,05\%, obtenidas para An. darlingien Villavicencio. A partir de estos datos, se estableció que una persona recibe al año 6.205 picaduras de An. darlingi (17 picaduras por persona por noche multiplicado por 365 días). Para calcular el número de picaduras infecciosas recibidas por una persona en un año, se tomó la cifra de 6.205 picaduras por persona por año y se multiplicó por la proporción de mosquitos de An. darlingi infectados (1/2.125), lo que dio como resultado que una persona puede recibir 2,9 picaduras infecciosas por año.

\section{Discusión}

Los estudios entomológicos en el área periurbana de Villavicencio, donde se presenta transmisión de paludismo, permitieron establecer que $A n$. darlingi, considerado el vector principal de esta enfermedad en la región amazónica $(1,2,4)$, fue la especie más abundante en las capturas con atrayente humano. Se encontró en diversos sitios de cría, que incluyeron fuentes de agua naturales y otros depósitos hechos por el hombre, los cuales en ocasiones estuvieron completamente expuestos al sol. Sin embargo, la vegetación circundante o emergente siempre estuvo presente, proporcionando el refugio necesario que asegura la supervivencia de las larvas hasta la emergencia de los adultos (35). Estas observaciones permitieron confirmar la capacidad de adaptación de An. darlingi y la posibilidad que tiene de colonizar diferentes sitios de cría, característica que ha sido descrita para esta especie y que favorece su presencia y proliferación en ambientes modificados, lo que aumenta el riesgo de exposición de la población humana a su picadura $(15,18,36,37)$ cuando se dan los procesos de urbanización (38).

La caracterización del comportamiento de picadura de An. darlingipermitió confirmar el hábito exofágico y endofágico característico de esta especie $(5,6,17,39)$. Presentó actividad hematofágica durante toda la noche, con un valor máximo entre las 18:00 y las 19:00, patrón que ha sido observado en América, particularmente en Guyana Francesa, Mato Grosso, Rondonia y Roraima (Brasil) y el sureste de Amazonas $(8,16,40)$; sin embargo, las bajas densidades de An. darlingi registradas durante el estudio no permiten establecer si esta población presenta una mayor actividad en otra hora de la noche, como se ha descrito en otras poblaciones de la región amazónica.
Se estableció que está presente todo el año, con una densidad promedio de 17 mosquitos por persona por noche. La baja densidad registrada, característica propia de esta especie $(11,15)$, ya se había descrito en esta localidad (18). En América, las tasas de picadura de An. darlingi son variables, con valores que oscilan entre 0,08 y 1.443 mosquitos por persona por noche $(1,6,7,9,11,19,37,41)$. Sin embargo, se ha establecido que a pesar de las bajas densidades, esta especie es capaz de mantener la transmisión de malaria ya que puede infectarse con las diferentes especies de Plasmodium $(25,40)$.

La densidad de An. darlingi, observada en Villavicencio entre julio de 2008 y junio de 2009, exhibió un patrón de comportamiento monomodal con un aumento en febrero a valores entre 35 y 89 mosquitos por persona por noche. En los otros meses de estudio estuvo por debajo de 27,1 mosquitos por persona por noche y no se presentó una diferencia estadísticamente significativa entre los valores registrados en cada uno de ellos. Se observó que la población de An. darlingi aumentó cuando se registraron niveles de precipitación inferiores a $100 \mathrm{~mm}$, patrón de comportamiento que había sido descrito para esta especie en Venezuela, donde se observó una mayor abundancia durante los meses de menor precipitación (42). En los demás meses de estudio, no se observó un patrón de comportamiento que pueda ser explicado por los niveles de precipitación y la temperatura. En América, para esta especie, se han descrito diversos patrones de fluctuación estacional; sin embargo, en algunos estudios se ha demostrado que no existe correlación entre los niveles de precipitación y la densidad de las especies de Anopheles $(1,11)$. Entre los patrones descritos se tienen aquellos que establecen que las mayores densidades se observan en los meses lluviosos y, las menores, en los meses más secos $(2,6,8,10)$, los que determinan que el aumento en la densidad se da en los periodos de transición entre la estación seca y la lluviosa $(11,12,43)$. Sin embargo, se ha considerado que los cambios en la densidad de An. darlingi no solo depende de los factores antes mencionados, sino que responde a una interacción entre la disponibilidad de sitios de cría, el nivel de los ríos y otras variables ambientales $(15,16)$.

Un aspecto relevante del análisis del comportamiento de picadura de las especies de Anopheles, es determinar su relación con la actividad humana. A este respecto se observó que An. darlingi estuvo presente durante toda la noche dentro y fuera del 
domicilio humano, sin presentar diferencia en la actividad de picadura entre estos dos ambientes. A partir de este resultado se puede establecer que la población humana tiene el mismo riesgo de contacto con este vector en los dos lugares. El nivel de exposición a la picadura se ve incrementado porque las personas están activas entre las $18: 00$ y las 22:00 en el intradomicilio y peridomicilio, y entre las 04:00 y las 06:00, en el intradomicilio, que corresponde con el P1 y el P3 cuando se registraron los mayores porcentajes de contribución observados para An. darlingi. Se debe anotar que el P1 coincide con los mayores valores de actividad horaria de picadura exhibidos por $A n$. darlingi; sin embargo, esto no ocurre durante el P3. Según estos resultados, es necesario promover la utilización de alternativas de control, como uso de repelentes, que reduzcan el contacto con el vector cuando la población está activa, dado que se observó que durante el P1 llegan al intradomicilio mosquitos infectados con Plasmodium spp. Con respecto al periodo entre las 22:00 y las 04:00, se observó que había un aumento en la proporción de mosquitos en el intradomicilio comparado con el peridomicilio, aspecto que confirma que el uso de mosquiteros puede reducir la probabilidad de contacto de la población con An. darlingi.

Anopheles darlingi fue la única especie en la que se detectó infección con $P$. falciparum en el área de estudio. La tasa de infección de 0,05\% es similar a la encontrada en un estudio previo que incluyó mosquitos de este departamento, en el que se estimó una tasa de infección con el mismo parásito de 0,07\% (44). En los estudios de zonas periurbanas de Puerto Carreño en Colombia, y Rondonia y Roraima en Brasil, con características de urbanización similares a las incluidas en este estudio, se encontró que las tasas de infección para An. darlingi pueden ser variables, con valores entre $0,008 \%$ y $8,5 \%(19,40)$.

Se estimó para Villavicencio una tasa de inoculación entomológica de 2,9 picaduras infecciosas por persona por año. Este valor está dentro de los obtenidos en otras área de transmisión de malaria en América, 1,6 y 10 picaduras infecciosas por persona por año en Colombia y Brasil, 21,6 picaduras infecciosas por persona por año en la Amazonia de Guyana Francesa y 129 picaduras infecciosas por persona por año en el sureste de Venezuela $(1,2,19,37,45)$. Particularmente, en las zonas periurbanas de Colombia y Brasil, se registran tasas de inoculación entomológica entre
1,6 y 50,0 picaduras infecciosas por persona por año, respectivamente $(19,40)$.

Al observar la distribución de los casos de paludismo durante 2008 y 2009 en Villavicencio, se estableció que el aumento de los casos se dio en los meses cuando se registra un incremento en la densidad de An. darlingi. Esta misma tendencia se observó en la región sur de Venezuela, donde se encontró que existe una asociación entre la abundancia y la incidencia mensual de la malaria (1).

Se confirma la importancia que tiene An. darlingi en el mantenimiento de la transmisión de malaria en Villavicencio a pesar de la baja densidad y tasa de infección. Se estableció que la disponibilidad de sitios de cría, cuando se reducen los niveles de precipitación, favorece la permanencia de esta especie a lo largo del año, haciendo de la zona periurbana de Villavicencio un área vulnerable a la aparición de casos de malaria de origen autóctono o cuando se introducen portadores de parásitos, condición que se comparte con otras áreas de transmisión periurbana de paludismo en América $(18,24)$.

Teniendo en cuenta los patrones de comportamiento de picadura observados para An. darlingi, se sugiere complementar las medidas intradomiciliarias de control para malaria, como, por ejemplo, el uso de mosquiteros tratados con piretroides, con medidas de protección personal que reduzcan el contacto entre humano y vector en la horas en que la población está más expuesta a las picaduras de los mosquitos.

\section{Agradecimientos}

Los autores manifestamos agradecimiento a Jaime Ramírez, Marco Fidel Suárez, Armando Escobar, Ruth Mary Benavides y Nelson Peña, por su apoyo en la realización del trabajo de campo.

\section{Conflicto de intereses}

Los autores declaramos no tener conflicto de intereses.

\section{Financiación}

Colciencias, Proyecto "Determinación del papel como vectores de malaria de las especies de Anopheles en tres regiones de Colombia" (Código: 1101-343-19196); División de Investigación sede Bogotá (DIB), Universidad Nacional de Colombia Proyecto "Determinación del papel como vectores de malaria de las especies de Anopheles en el departamento del Meta, Colombia"; Código Quipu: 
20201009479, Instituto Nacional de Salud y Secretaría de Salud del Meta.

\section{Referencias}

1. Magris M, Rubio-Palis $\mathbf{Y}$, Menares C, Villegas L. Vector bionomics and malaria transmission in the Upper Orinoco River Southern Venezuela. Mem Inst Oswaldo Cruz.2007;102:303-11.http://dx.doi.org/10.1590/S007402762007005000049

2. Girod R, Gaborit P, Carinci R, Issaly, Fouque F. Anopheles darlingi bionomics and transmission of Plasmodium falciparum, Plasmodium vivax and Plasmodium malariae in Amerindian villages of the Upper-Maroni Amazomian forest, French Guiana. Mem Inst Oswaldo Cruz. 2008;103:702-10. http://dx.doi.org/10.1590/S0074-02762008000700013

3. Sinka ME, Rubio-Palis Y, Manguin S, Patil AP, Temperley WH, Gething PW, et al. The dominant Anopheles vectors of human malaria in the Americas: Occurrence data, distribution maps and bionomic précis. Parasit Vectors. 2010;3:72. http://dx.doi.org/10.1186/1756-3305-3-72.

4. Hiwat H, Bretas G. Ecology of Anopheles darlingi Root with respect to vector importance: A review. Parasit Vectors. 2011;4:177. http://dx.doi.org/10.1186/1756-3305-4-177.

5. Forattini OP. Comportamento exofilo de Anopheles darlingi Root, em regiao meridional do Brasil. Rev Saúde Públ.1987;21:291-304.http://dx.doi.org/10.1590/S003489101987000400002

6. Rubio-Palis Y. Observaciones sobre el patrón de actividad hematofágica del vector de la malaria Anopheles darlingi en las poblaciones del sur de Venezuela. Boletín de la Dirección de Malariología y Saneamiento Ambiental. 1995;35:66-70.

7. Rozendaal JA. Biting and resting of Anophleles darling in the Surinam rainforest. J Am Mosq Control Assoc. 1989;5:351-8.

8. Voorham J. Intra-population plasticity of Anopheles darlingi (Diptera: Culicidae) biting activity patterns in the state of Amapa, Brazil. Rev Saúde Públ. 2002;36:75-80. http:// dx.doi.org/10.1590/S0034-89102002000100012

9. León W, Valle J, Naupay R, Tineo E, Rosas A, Palomino M. Comportamiento estacional de Anopheles (Nyssorhynchus) darlingi Root, 1926 en localidades de Loreto y Madre de Dios, Perú 1999-2000. Rev Peru Med Exp Salud Pública. 2003;20:22-7.

10. Tineo E, Medina A, Fallaque C, Chávez L, Quispe S, Mercado M, et al. Distribución geográfica y comportamiento estacional de Anopheles (Nyssorhynchus) darlingi Root, 1926, en localidades de la frontera Perú-Bolivia, Madre de Dios, Perú. Rev Peru Med Exp Salud Pública. 2003; 20:78-83.

11. Moreno JE, Rubio-Palis Y, Páez E, Pérez E, Sánches V. Abundance, biting behaviour and parous rate of anopheline mosquito species in relation to malaria incidence in goldmining areas of southern Venezuela. Med Vet Entomol. 2007;21:339-49.http://dx.doi.org/10.1111/j.1365-2915 .2007.00704.x

12. Achee N, Grieco J, Rejmankova E, André R, Vanzie E, Polanco $\mathrm{J}$, et al. Biting pattern and seasonal densities of Anopheles mosquitoes in the Cayo District, Belice, Central America with emphasis in Anopheles darlingi. $\mathrm{J}$
Vector Ecol. 2006;31:45-57.http://dx.doi.org/10.3376/10811710(2006)31[45:BPASDO]2.0.CO;2

13. Renjifo S. Disecciones de mosquitos del género Anopheles en la Intendencia del Meta. Caldasia. 1948;5:99-103.

14. Renjifo S, Zulueta J. Five years observations of rural malaria in Eastern Colombia. Cespedecia. 1974;9-12:305-22.

15. Montoya-Lerma J, Solarte YA, Giraldo-Calderon GI, Quiñones ML, Ruiz-Lopez F, Wilkerson RC, et al. Malaria vector species in Colombia - A review. Mem Inst Oswaldo Cruz. 2011;106(Supl.1):223-38. http://dx.doi.org/10.1590/ S0074-02762011000900028

16. Charlwood JD. Biological variation in Anopheles darlingi Root 1926. Mem Inst Oswaldo Cruz. 1996;91:391-8. http:// dx.doi.org/10.1590/S0074-02761996000400001

17. Elliott R. The influence of vector behaviour on malaria transmission. Am J Trop Med Hyg. 1972;21:765-3.

18. Brochero H, Rey G, Buitrago LS, Olano VA. Biting activity and breeding sites of Anopheles species in the municipality Villavicencio, Meta, Colombia. J Am Mosq Control. 2005;21:182-6.

19. Jiménez P, Conn J, Witz R, Brochero H. Anopheles (Díptera: Culicidae) vectores de malaria en el municipio de Puerto Carreño, Vichada, Colombia. Biomédica. 2012;32(Supl.1):13-21.

20. Zambrano P. Informe final de malaria, semanas 1 a 52 Colombia 2005. Inf Quinc Epidemiol Nac. 2006;11:49-53.

21. Padilla JC, Álvarez G, Montoya R, Chaparro P, Herrera S. Epidemiology and control of malaria in Colombia. Mem Inst Oswaldo Cruz. 2011;106:114-22. http://dx.doi.org/10.1590/ S0074-02762011000900015

22. Instituto Nacional de Salud. Sistema Nacional de Vigilancia en Salud Pública (SIVIGILA) 2000-2011. Bogotá D.C.: INS; 2011

23. Carrasquilla $G$, Banguero $M$, Sánchez $P$, Carvajal $F$, Barker RH jr, Gervais GW, et al. Epidemiologic tools for malaria surveillance in an urban setting of low endemicity along the Colombian Pacific Coast. Am J Trop Med Hyg. 2000;62:132-7.

24. Ochoa J. Osorio L. Epidemiology of urban malaria in Quibdó, Chocó. Biomédica. 2006;26:278-85.

25. Deane LM. Malaria vector in Brazil. Mem Inst Oswaldo Cruz. 1986;81:5-14.

26. IGAC, Instituto Geográfico Agustín Codazzi. Diccionario Geográfico de Colombia. Tercera edición. Bogotá: Instituto Geográfico Agustín Codazzi; 1996.

27. Departamento Administrativo Nacional de Estadística (DANE). Evidencia reciente del comportamiento de la migración interna en Colombia a partir de la encuesta continua de hogares. Fecha de consulta: 17 de septiembre de 2012. Disponible en: http://www. http://www.dane.gov.co/ files/banco_datos/Migracion/migracion_interna_Clbia.pdf.

28. Linthicum KJ. A revision of the Argyritarsis section of the subgenus Nyssorhynchus of Anopheles (Diptera:Culicidae). Mosq Sys. 1988;20:98-271.

29. Faran ME. A revision of the Albimanus section of the subgenus Nyssorhynchus. Contrib Am Entomol Inst. 1980;15:1-215. 
30. Faran ME, Linthicum KJ. A handbook of the Amazonian species of Anopheles (Nyssorhynchus) (Diptera: Culicidae). Mosq Syst. 1981;13:1-81.

31. González R, Carrejo N. Introducción al estudio taxonómico de Anopheles de Colombia claves taxonómicas y notas de distribución. Segunda edición. Cali: Programa Editorial de la Universidad del Valle; 2009.

32. Wirtz R, Burkot T, Andre RG, Rosenberg R, Collins WE, Roberts DR. Identification of Plasmodium vivax sporozoites in mosquitoes using an enzyme linked immunosorbent assay. Am J Trop Med Hyg. 1985;54:1048-54.

33. Wirtz R, Burkot T, Graves T, Andre RG. Field evaluation of enzyme-linked immunosorbent assays for Plasmodium falciparum and Plasmodium vivax sporozoites in mosquitoe (Diptera: Culicidae) from Papua New Guinea. J Med Entomol. 1987;24:433-7.

34. Macdonald G. The epidemiology and control of malaria. Oxford: Oxford University Press; 1957.

35. Orr BK, Resh VH. Experimental test of the influence of aquatic macrophyte cover on the survival of Anopheles larvae. J Am Mosq Contr Assoc. 1989;5:581-5.

36. Pinedo-Cancino V, Sheen P, Tarazona-Santos E, Oswald WD, Jeri C, Vittor AY, et al. Limited diversity of Anopheles darlingi in the peruvian amazon region of lquitos. Am J Trp Med Hyg. 2006;75:238-45.

37. Vittor AY, Gilman RH, Tielsch J, Glass G, Shields T, Lozano WS. The effect of deforestation on the humanbiting rate of Anopheles darlingi, the primary vector of $P$. falciparum malaria in the Peruvian Amazon. Am J Trop Med Hyg. 2006;74:3-11.

38. Gil LH, Tada MS, Katsuragawa TH, Ribolla PE, Silva LH. Urban and suburban malaria in Rondonia (Brazilian Western Amazon) II: Perennial transmissions with high anopheline densities are associated with human environmental changes. Mem Inst Oswaldo Cruz. 2007;102:271-6. http:// dx.doi.org/10.1590/S0074-02762007005000013

39. Charlwood JD. Observation of the bionomics of Anopheles darlingi Root (Diptera: Culicidae) from Brazil. Bull Entomol Res. 1980;70:685-92.

40. Da Silva-Vasconcelos A, Kató MY, Mourão EN, de Souza RT, Lacerda RN, Sibajev A, et al. Biting indices, hostseeking activity and natural infection rates of anopheline species in Boa Vista, Roraima, Brazil from 1996 to 1998. Mem Inst Oswaldo Cruz. 2002;97:151-61. http://dx.doi.org/10. 1590/S0074-02762002000200002

41. Hiwat H, Issaly J, Gaborit P, Somai A, Samjhawan A, Sardjoe P, et al. Behavioral heterogeneity of Anopheles darlingi (Diptera: Culicidae) and malaria transmission dynamics along the Maroni River, Suriname, French Guiana. Trans R Soc Trop Med Hyg. 2010;104:207-13. http://dx.doi. org/10.1016/j.trstmh.2009.07.007

42. Rubio-Palis Y. Anopheles (Nyssorhynchus) de Venezuela: taxonomía, bionomía, ecología e importancia médica. Maracay, Venezuela: Escuela de Malariología y Saneamiento Ambiental; 2000.

43. Povoa MM, Wirtz RA, Lacerda R, Miles MA, Warhurst D. Malaria vectors in the municipality of Serra do Navio, State of Amapá, Amazon Region, Brazil. Mem Inst Oswaldo Cruz.2001;96:179-84.http://dx.doi.org/10.1590/S007402762001000200008

44. Herrera S, Suárez M, Sánchez G, Quiñones ML, Herrera M. Uso de la técnica inmunorradiométrica (IRMA) en Anopheles de Colombia para la identificación de esporozoítos de Plasmodium. Colombia Médica. 1987;18:2-6.

45. Soares Gil LH, Alves FP, Zieler H, Salcedo JMV, Durlacher RR, Cunha RPA, et al. Seasonal malaria transmission and variation of anopheline density in two distinct endemic areas in Brazilian Amazonia. J Med Entomol. 2003;40:636-41. 